\title{
Entanglement Genesis by Ancilla-Based Parity Measurement in 2D Circuit QED
}

\author{
O.-P. Saira, J. P. Groen, J. Cramer, M. Meretska, G. de Lange, and L. DiCarlo \\ Kavli Institute of Nanoscience, Delft University of Technology, Post Office Box 5046, 2600 GA Delft, The Netherlands
}

(Received 22 November 2013; published 20 February 2014)

\begin{abstract}
We present an indirect two-qubit parity meter in planar circuit quantum electrodynamics, realized by discrete interaction with an ancilla and a subsequent projective ancilla measurement with a dedicated, dispersively coupled resonator. Quantum process tomography and successful entanglement by measurement demonstrate that the meter is intrinsically quantum nondemolition. Separate interaction and measurement steps allow the execution of subsequent data-qubit operations in parallel with ancilla measurement, offering time savings over continuous schemes.
\end{abstract}

Controlling the entanglement between qubits is central to the development of every quantum computing architecture. Early efforts with superconducting quantum circuits relied on quantum interference for this purpose. Programmed sequences of one- and few-qubit gates fitting within qubit coherence times have allowed the generation of two- and three-qubit entanglement [1-4] and the implementation of elementary quantum algorithms [5-9] and games [10].

Recently, focus has shifted toward generating and preserving entanglement by nondemolition measurement of multiqubit observables and their use in feedback loops as required for quantum error correction [11]. Of particular interest is the parity measurement [12-14] that discriminates between states in a multiqubit register with even or odd total excitation number. Parity measurement on four data qubits at the corners of every square tile on a lattice is needed to realize surface codes, offering the highest faulttolerance thresholds to date $[15,16]$.

A convenient approach to implementing a parity measurement is a two-step indirect scheme involving coherent interaction of the data qubits with an ancillary qubit and subsequent strong measurement of this ancilla. To date, indirect four-qubit parity measurements have been achieved only in trapped-ion systems [17]. In the solid state, parity measurement using an ancillary electron spin has been used to generate probabilistic entanglement between two nuclear spins in nitrogen-vacancy centers in diamond [18]. More recently, parity measurement of two transmon qubits using a dispersively coupled 3D cavity has been used in a digital feedback loop to generate entanglement deterministically [19]. An important next step is the realization of parity measurements in an architecture amenable to surface coding.

In this Letter, we present an ancilla-based two-qubit parity measurement in a planar circuit QED (cQED) architecture [20]. Tomographic characterization shows that dephasing within even and odd parity subspaces is due to intrinsic qubit decoherence during interaction and measurement steps, making the parity meter intrinsically quantum nondemolition (QND). As a further demonstration of this nondemolition character, we generate entanglement by parity measurement on a maximal superposition state. Performing all tomographic data-qubit operations after the ancilla measurement, we achieve a concurrence of $0.46(0.38)$ in the even (odd) measurement outcome using a single threshold for conditioning on the ancilla readout, matching the open-loop performance of the recent implementation based on continuous measurement [19]. A distinct architectural advantage of our two-step scheme is the possibility to continue operations on the data qubits while the ancilla measurement is performed. Performing the entanglement-by-measurement protocol using such parallel timing instead, the concurrence in the even (odd) parity outcome improves to $0.74(0.63)$.

Our quantum processor, shown in Fig. 1(a), combines four transmon qubits (data qubits $D_{1}$ and $D_{2}$, ancilla $A$, and a fourth unused qubit) and five resonators, expanding the architecture introduced in Ref. [21]. A high- $Q$ resonator bus $(B)$ couples to every qubit and mediates all interactions. Dedicated resonators, each dispersively coupled to one qubit, allow frequency-multiplexed individual qubit readouts via a common feedline [22,23]. Finally, flux-bias lines allow individual tuning of qubit transition frequencies with $1 \mathrm{~ns}$ resolution [5].

The interaction step of the parity meter involves two controlled-phase (C-PHASE) gates between $A$ and the data qubits. We compile these gates using a toolbox of resonant qubit-bus interactions proposed in Ref. [24] and first realized with phase qubits [25]. A map of coherent qubit-bus interactions in the one- and two-excitation manifolds is obtained by varying the duration and amplitude of a flux pulse on $D_{1}$ starting from $\left|e_{1}, 0\right\rangle$ and $\left|e_{1}, 1\right\rangle$, respectively [Fig. 1(b)]. We use $\left|g_{i}\right\rangle,\left|e_{i}\right\rangle$, and $\left|f_{i}\right\rangle$ to denote the ground, first, and second excited states of transmon $i$, respectively, and $|n\rangle$ to refer to the $n$-photon state of the bus. A half-period of oscillation at the $\left|e_{1}, 0\right\rangle \leftrightarrow\left|g_{1}, 1\right\rangle$ resonance [Fig. 1(c)] implements an isWAP gate [26] between $D_{1}$ and $B$. A full period at the $\left|f_{1}, 0\right\rangle \leftrightarrow\left|e_{1}, 1\right\rangle$ 


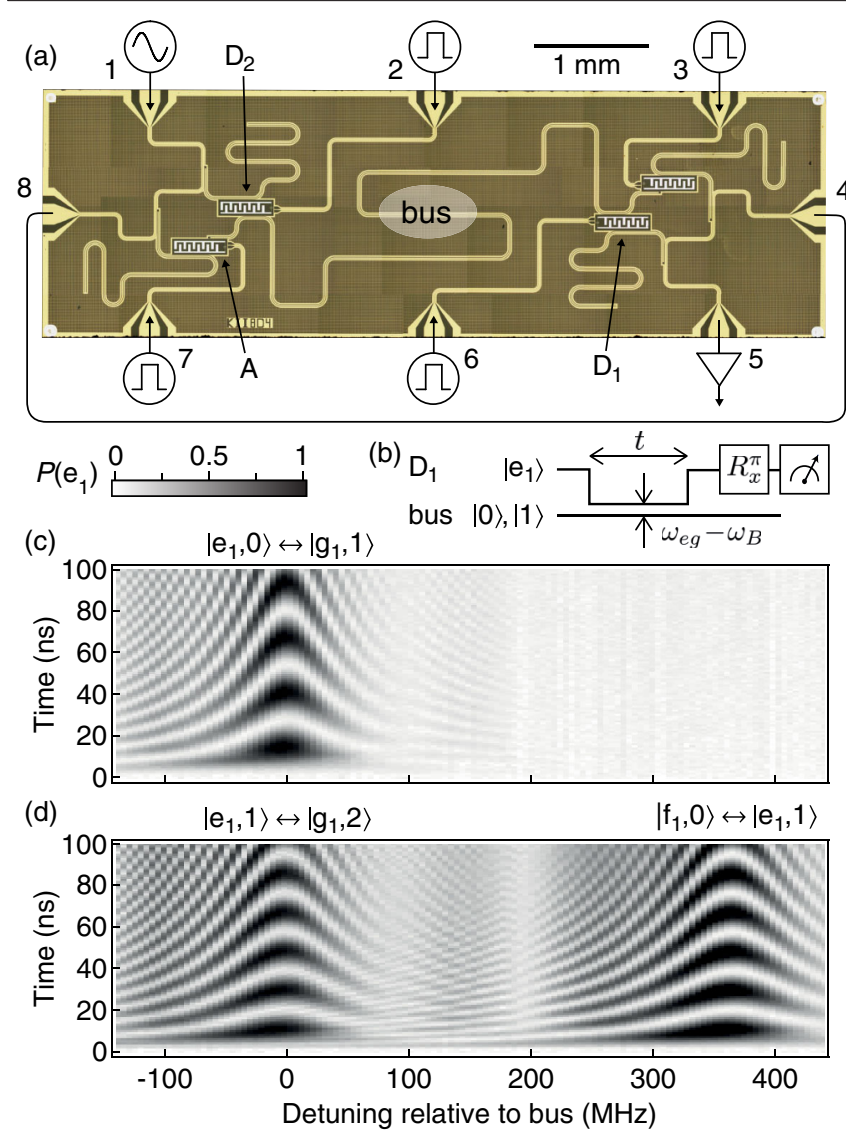

FIG. 1 (color online). (a) cQED processor with four transmons ( $A, D_{1}, D_{2}$, and an unused one at top right) coupled to a bus resonator. Each transmon has a dedicated readout resonator that is addressed through the shared feedline (ports 1-8-4-5) and a local flux-bias line (ports $2,3,6,7$ ) that allows tuning of the transition frequencies with $\sim 1 \mathrm{~ns}$ resolution [5]. A coaxial cable connects ports 4 and 8 off the chip. (b) Gate sequence for coherent swapping of excitations between $D_{1}$ and the bus by nonadiabatic qubit tuning. (c),(d) Measured average qubit populations at the end of the sequence for the one- and two-excitation manifolds, respectively. An excitation can be swapped from $D_{1}$ to the bus (or vice versa) in $13.1 \mathrm{~ns}$ when the $g_{1}-e_{1}$ transition is resonant with $B$. (d) In the two-excitation manifold, population transfer occurs when either the $g_{1}-e_{1}$ or the $e_{1}-f_{1}$ transition is resonant with $B$ with a half-period of 9.3 ns. The ratio of the measured periods agrees with the $\sqrt{2}$ enhancement of the effective coupling predicted by theory.

resonance in the two-excitation manifold [Fig. 1(d)] implements a C-PHASE gate [27]. We implement C-PHASE gates between $A$ and $D_{i}$ using three qubit-bus primitives: $i \mathrm{SWAP}_{A, B} \mathrm{C}-\mathrm{PHASE}_{B, D_{i}}$, and $i \mathrm{SWAP}_{A, B}$. Note that the $n$ C-PHASE gates in the interaction step of an $n$-qubit ancillabased parity measurement can be realized with only $n+2$ qubit-bus primitives, since back-to-back $A-B$ swaps can be compiled away ( $n=2$ here).

The ideal projective ancilla measurement comprising the second step of the parity meter is high fidelity, fast relative to intrinsic qubit decoherence, and does not impose any
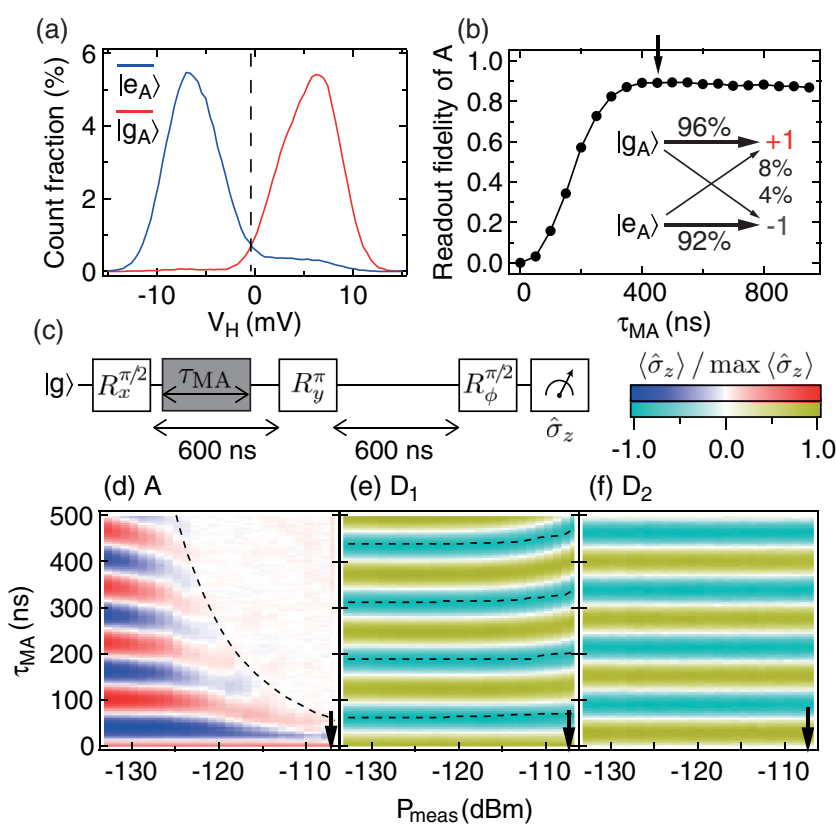

FIG. 2 (color online). (a) Histograms of $V_{H, A}\left(\tau_{\mathrm{MA}}=450 \mathrm{~ns}\right)$ for computational states of $A$. The dashed line is the fidelity maximizing digitizing threshold. (b) Single-shot ancilla readout fidelity $\mathcal{F}_{A}$ as a function of the measurement pulse duration $\tau_{\mathrm{MA}}$. Maximal $\mathcal{F}_{A}=$ $89 \%$ is attained at $\tau_{\mathrm{MA}}=450 \mathrm{~ns}$ (arrow). Inset: Corresponding calibrated readout error model. (c) Gate sequence used to study qubit dephasing induced by $A$ measurement. A readout pulse of duration $\tau_{\mathrm{MA}}$ and power $P_{\text {meas }}$ is embedded in a fixed-length echo sequence performed on qubit (d) $A$, (e) $D_{1}$, and (f) $D_{2}$. The azimuthal angle $\phi$ of the final $\pi / 2$ rotation is swept from 0 to $8 \pi$ jointly with $\tau_{\mathrm{MA}}$ to facilitate discerning deterministic phase shifts and dephasing. The plots show averaged $\hat{Z}$ measurements normalized to compensate for the fixed loss of contrast due to intrinsic decoherence. In (d), the dashed line is a theoretical prediction for $98 \%$ loss in phase contrast. Dashed lines in (e) are equal-phase contours accounting for ac Stark shift on $D_{1}$. All theory curves were calculated using measured parameters [28]. (f) No effect on $D_{2}$ is observed. Arrows indicate the power used for $A$ readout in Figs. 3 and 4. The incident power corresponding to the one-photon average population in the $A$ readout resonator is $-133 \mathrm{dBm}$.

additional backaction on data qubits. We probe the ancillastate-dependent transmission of a dedicated, dispersively coupled resonator [28] with a microwave pulse applied to the feedline near the resonator's fundamental $(7.366 \mathrm{GHz})$. Following increasingly standard practice in cQED [29], we use a Josephson parametric amplifier (JPA) at the front end of the amplification chain to boost the readout fidelity and reduce the pulse duration [30]. Histograms of the integrated homodyne voltage $V_{H, A}$ with an ancilla prepared in $\left|e_{A}\right\rangle$ and $\left|g_{A}\right\rangle$ reveal an optimal single-shot fidelity of $89 \%$ at measurement pulse duration $\tau_{\mathrm{MA}}=450 \mathrm{~ns}$ while probing with $\sim 400$ intraresonator steady-state photons [Figs. 2(a) and 2(b)]. Crucially, the ancilla measurement does not induce any significant dephasing on data qubits, despite the high level of measurement power used. To show this, we embed ancilla readout pulses in the first half of standard 
echo experiments on $A, D_{1}$, and $D_{2}$ [Figs. 2(c)-2(f)]. For $A$, the expected coherence loss due to measurement is observed at all readout powers. For $D_{1}\left(D_{2}\right)$, only $2.5 \% \pm$ $1 \%(2.3 \% \pm 1 \%)$ of contrast is lost at the chosen measurement strength [28]. On $D_{1}$, whose readout resonator is closest in frequency to that of $A$, we observe powerdependent qubit detuning consistent with the ac Stark shift [31]. We correct the induced deterministic phase either with a $5 \mathrm{~ns}$ detuning flux pulse or in postprocessing. To completely test the QND character of ancilla measurement, we perform quantum process tomography on the data qubits undergoing $326 \mathrm{~ns}$ of idling, with and without an applied $300 \mathrm{~ns}$ ancilla readout pulse [28]. The 0.97 process fidelity between these two processes, after correcting for the phase accrued by $D_{1}$ with measurement on, confirms the low level of backaction.

We now combine the interaction and measurement steps described into the full parity measurement protocol shown in Fig. 3(a). We first quantify the parity measurement fidelity by analyzing the correlation between measurement results $\mathcal{M}_{P}= \pm 1$ for data-qubit input states of definite parity, namely, the four computational states. The optimal digitizing threshold maximizes the parity readout fidelity at $\mathcal{F}_{P}=69 \%$ [Fig. 3(b)]. To test the meter's ability to preserve (suppress) coherence within (across) parity subspaces, we apply parity measurement to the maximal but separable superposition state $\frac{1}{2}\left(\left|g_{1} g_{2}\right\rangle+\left|g_{1} e_{2}\right\rangle+\left|e_{1} g_{2}\right\rangle+\right.$ $\left.\left|e_{1} e_{2}\right\rangle\right)$ created using two $\pi / 2$ pulses. State tomography of the data qubits at the end of the interaction step [parallel timing, Fig. 3(c)] shows that the average absolute coherence between states of different parity $\left[\left(\left|\rho_{g e, g g}\right|+\left|\rho_{g e, e e}\right|+\right.\right.$ $\left.\left|\rho_{\text {eg,gg }}\right|+\left|\rho_{\text {eg,ee }}\right|\right) / 4$, where $\left.\rho_{i j, k l}=\left\langle i_{1} j_{2}|\rho| k_{1} l_{2}\right\rangle\right]$ is suppressed by $90 \% \pm 1 \%$, while the average intraparity absolute coherence $\left(\left|\rho_{e e, g g}\right|+\left|\rho_{e g, g e}\right|\right) / 2$ decreases only $10 \% \pm 1 \%$. Similarly, state tomography at the end of the measurement step (serial timing) shows a total intraparity coherence loss of $32 \% \pm 1 \%$, consistent with intrinsic qubit decoherence during $\tau_{\mathrm{MA}}$. For parallel timing, conditioning on $\mathcal{M}_{P}=+1(-1)$ unveils highly entangled states with concurrence 0.74 (0.63) and Bell-state fidelity $87 \%(81 \%)$. The corresponding density matrices are shown in Figs. 3(d) and 3(e), respectively. For serial timing, these values reduce to $0.46(0.38)$ and $73 \%(67 \%)$, respectively.

Quantum process tomography of the data qubits with and without conditioning on the $\mathcal{M}_{P}$ outcome [28] provides the most complete characterization of the parity measurement. For parallel timing, the fidelities to the corresponding ideal process are $0.91,0.84$, and 0.79 for no $\mathcal{M}_{P}$ conditioning, conditioning on $\mathcal{M}_{P}=+1$, and conditioning on $\mathcal{M}_{P}=-1$, respectively. For serial timing, the respective process fidelities are $0.77,0.70$, and 0.65 . From the process tomograms, we determined that the dominant error in the coherent interaction step was the $89 \%$ population transfer efficiency of the isWAP gate between ancilla and bus [32].

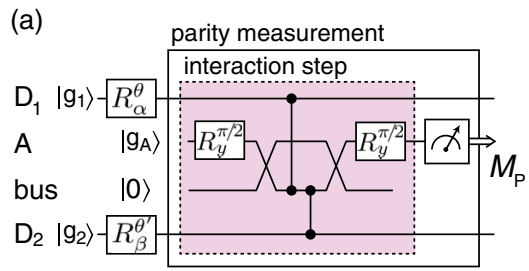

(b) $\left|\bar{g}_{1} g_{2}\right\rangle\left|\bar{e}_{1} e_{2}\right\rangle\left|\bar{e}_{1} g_{2}\right\rangle\left|\bar{g}_{1} e_{2}\right\rangle$

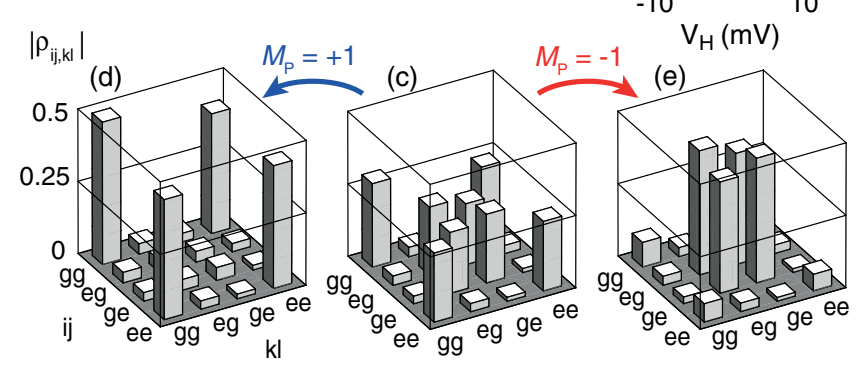

FIG. 3 (color online). (a) Gate sequence realizing an indirect parity measurement of $D_{1}$ and $D_{2}$ using coherent interactions with $A$ and a subsequent projective measurement of $A$. (b) Histograms of the integrated voltage $V_{H, A}\left(\tau_{\mathrm{MA}}=450 \mathrm{~ns}\right)$ for computational basis-state inputs prepared using initial rotations $R_{\alpha}^{\theta}, R_{\beta}^{\theta^{\prime}} \in\left\{I, R_{x}^{\pi}\right\}$. The digitizing threshold indicated by the dashed line produces the maximum parity fidelity $\mathcal{F}_{P}=69 \%$. (c)-(e) Manhattan-style plots of data-qubit density matrices after the parity measurement for the separable superposition input state prepared with $R_{\alpha}^{\theta}=R_{\beta}^{\theta^{\prime}}=R_{y}^{\pi / 2}$. (c) In the unconditioned tomogram, the density matrix elements indicative of coherences between the parity subspaces have been suppressed by $90 \%$ in magnitude. The spurious residual probability amplitudes can be attributed almost entirely to the infidelity of the two iswAP operations. (d),(e) The conditioned tomograms demonstrate probabilistic entanglement by measurement, reaching $87 \%$ $(81 \%)$ fidelity to the even (odd) Bell state and $0.74(0.63)$ concurrence for the even (odd) outcome. The higher fidelity of the even projection is in accordance with the error model of ancilla readout, where the dominating error mechanism is relaxation of $A$ during readout. For (c)-(e), the timing of tomographic prerotations and measurements corresponds to the parallel variation with $t_{\mathrm{MD}}=0$, as illustrated in Fig. 4(b).

Finally, we study the competition between parity readout fidelity and intrinsic qubit decoherence in the entanglement-by-measurement protocol. We vary the idling time $t_{\mathrm{MD}}$ between the end of the interaction step and the beginning of the data-qubit readout pulse for both serial and parallel timings [Figs. 4(a) and 4(b), respectively]. For serial timing [Fig. 4(c), open markers], we use $\tau_{\mathrm{MA}}=$ $t_{\mathrm{MD}}-50 \mathrm{~ns}$, resulting in a steep initial increase in concurrence owing to rapidly improving ancilla readout fidelity followed by a decay due to intrinsic data-qubit decoherence. To quantify the evolution from a product to an entangled state of data qubits, we consider Wootters's $\Lambda$ [33] used to define concurrence $C(\rho) \equiv \max \{\Lambda(\rho), 0\}$. Even though the initial maximal superposition state lies at the boundary between separable and entangled two-qubit states, decoherence in the data qubits pulls the state away from the boundary, as manifested by the negative 
(a) serial variation

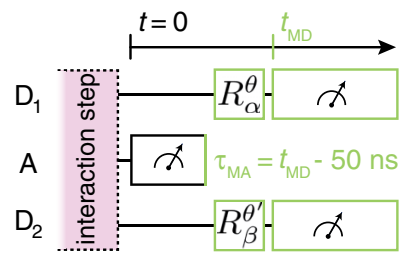

(b) parallel variation
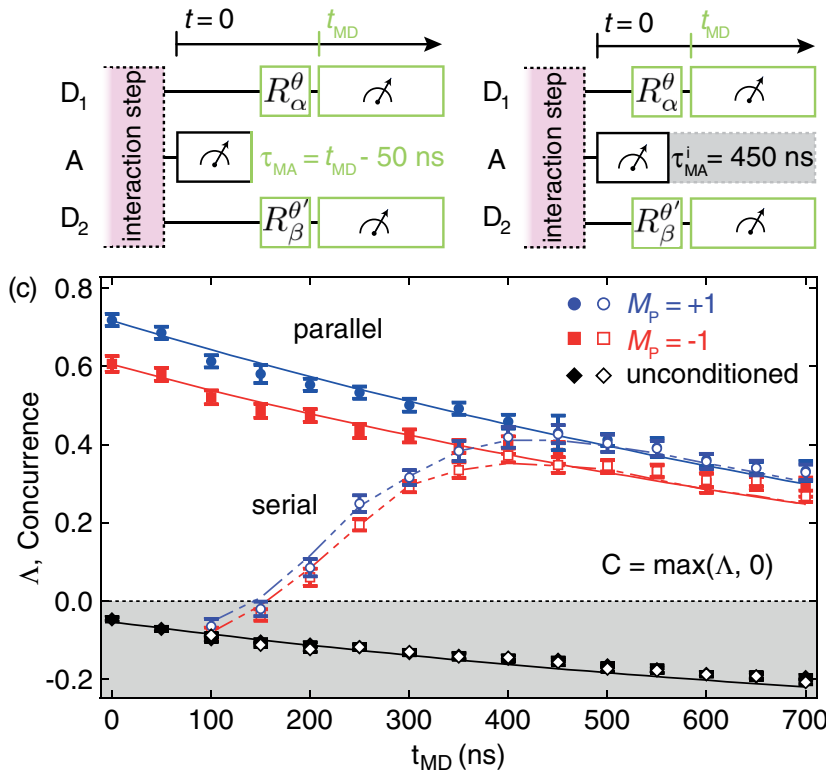

FIG. 4 (color online). Genesis and decay of two-qubit entanglement as a function of the time $t_{\mathrm{MD}}$ between the end of the interaction step and the beginning of the readout pulses on the data qubits. (a) Timing diagram for the serial variation, in which the ancilla measurement is completed strictly before operations on data qubits continue. (b) Timing diagram for the parallel variation, in which the duration $\tau_{\mathrm{MA}}$ of the ancilla readout pulse is constant at $1 \mu \mathrm{s}$. The homodyne voltage is integrated only during the first $\tau_{\mathrm{MA}}^{i}=450 \mathrm{~ns}$. The tomographic prerotations and the readout pulses for the data qubits can overlap with the ancilla readout. For $t_{\mathrm{MD}}=0$, the prerotations are done simultaneously with the ancilla $R_{y}^{\pi / 2}$ gate at the end of the interaction step. (a),(b) Elements drawn in green move together in time. (c) Wootters's $\Lambda$ [33] and concurrence $C=\max (\Lambda, 0)$ as a function of $t_{\mathrm{MD}}$ for the parallel and serial timings (filled and open markers, respectively) extracted from the two-qubit density matrix that is unconditioned (black diamonds), conditioned on the even outcome $\left(M_{P}=+1\right.$, blue circles), and conditioned on the odd outcome $\left(M_{P}=-1\right.$, red squares) of the ancilla measurement. Solid curves are based on a model that includes single-qubit relaxation and dephasing processes and uses the experimental density matrix at $t=0$ as the initial value. For the dashed curves, mixing of the parity subspaces according to calibrated readout errors is explicitly included.

$\Lambda$ observed without conditioning on the ancilla measurement [Fig. 4(c), black diamonds]. This pull imposes a minimum threshold in the ancilla readout fidelity to generate entanglement by conditioning on the measurement outcome. Entanglement is established after $\tau_{\mathrm{MA}} \approx 100 \mathrm{~ns}$. This time is quantitatively matched by a model including the calibrated ancilla readout errors. For parallel timing [Fig. 4(c), filled markers], in which we use the optimal integration time $\tau_{\mathrm{MA}}^{i}=450 \mathrm{~ns}$ for all $t_{\mathrm{MD}}$, entanglement decreases monotonically and consistently with the intrinsic qubit decoherence. For $t_{\mathrm{MD}}>450 \mathrm{~ns}$, the parallel and serial timings perform similarly because the ancilla readout fidelity is nearly constant.
The above entanglement by measurement is a discretized version of the continuous-time scheme investigated theoretically in Ref. [34]. The finite time to entanglement observed in serial timing is reminiscent of the entanglement genesis time required under continuous parity measurement. However, while the continuous scheme produces entanglement even starting from a maximally mixed state owing to the interplay of simultaneous Hamiltonian and measurement dynamics, entanglement by a discrete, projective parity measurement necessitates an initial superposition state of the data qubits. Instead, performing two parity measurements with single-qubit rotations in between would realize a QND Bell-state measurement [35], producing entanglement for any input two-qubit state. This protocol could be conveniently implemented with this processor in parallel timing by employing the unused qubit as a second ancilla.

In conclusion, we have realized a two-qubit parity meter in 2D cQED using a two-step scheme involving interaction of the data qubits with an ancilla and subsequent ancilla projection. The interaction step, employing resonant interactions at the raw speed set by qubit-bus coupling, can be efficiently compiled into $n+2$ primitives for $n$-qubit parity measurement. Detailed characterization of the ancilla readout performed via a dedicated dispersively coupled resonator demonstrates minimal measurement-induced dephasing of data qubits $(97 \%$ of single-qubit coherence retained), low measurement cross talk (2\% during simultaneous three-qubit readout) [28], and high single-shot fidelity (89\%). Applying the parity measurement on an unentangled superposition state of the two data qubits generates entanglement for both measurement outcomes, in both serial and parallel timings. In the former, we observe entanglement genesis after a $100 \mathrm{~ns}$ ancilla measurement. As a possible follow-up experiment, coupling a fifth qubit to the bus would allow the implementation of the four-qubit parity measurements necessary for quantum error correction using surface codes. We anticipate that the enhanced $2 \mathrm{D}+$ connectivity offered by recent fabrication developments $[36,37]$ will also allow the implementation of larger fragments of error-correcting lattices using this architecture.

O.-P. S. designed and fabricated the processor based on earlier devices by J. P. G. and M. M., did the measurements and data analysis, and wrote the manuscript with L. D. C. J. C. and J. P. G. devised the two-qubit C-PHASE tuneup. G.d.L. designed the mechanical parts used in the low-temperature setup. L. D. C. initiated and supervised the project. We thank W. Kindel and K. W. Lehnert for the JPA, D. Thoen and T. M. Klapwijk for NbTiN thin films, D. Ristè for assistance with measurements, and A.N. Jordan for discussions. We acknowledge funding from the Netherlands Organization for Scientific Research (NWO, VIDI scheme) and the EU FP7 Projects SOLID and SCALEQIT. 
Note added in proof.-Following submission of this manuscript, we became aware of similar work at IBM [38].

[1] M. Steffen, M. Ansmann, R. C. Bialczak, N. Katz, E. Lucero, R. McDermott, M. Neeley, E. M. Weig, A. N. Cleland, J. M. Martinis, Science 313, 1423 (2006).

[2] M. Ansmann et al., Nature (London) 461, 504 (2009).

[3] L. DiCarlo, M. D. Reed, L. Sun, B. R. Johnson, J. M. Chow, J. M. Gambetta, L. Frunzio, S. M. Girvin, M. H. Devoret, and R. J. Schoelkopf, Nature (London) 467, 574 (2010).

[4] M. Neeley et al., Nature (London) 467, 570 (2010).

[5] L. DiCarlo et al., Nature (London) 460, 240 (2009).

[6] T. Yamamoto et al., Phys. Rev. B 82, 184515 (2010).

[7] M. D. Reed, L. DiCarlo, S. E. Nigg, L. Sun, L. Frunzio, S. M. Girvin, and R. J. Schoelkopf, Nature (London) 482, 382 (2012).

[8] A. Dewes, R. Lauro, F. R. Ong, V. Schmitt, P. Milman, P. Bertet, D. Vion, and D. Esteve, Phys. Rev. B 85, 140503 (2012).

[9] E. Lucero et al., Nat. Phys. 8, 719 (2012).

[10] M. Mariantoni et al., Nat. Phys. 7, 287 (2011).

[11] M. H. Devoret and R. J. Schoelkopf, Science 339, 1169 (2013).

[12] R. Ruskov and A. N. Korotkov, Phys. Rev. B 67, 241305 (2003).

[13] K. Lalumière, J. M. Gambetta, and A. Blais, Phys. Rev. A 81, 040301 (2010); L. Tornberg and G. Johansson, Phys. Rev. A 82, 012329 (2010).

[14] D. DiVincenzo and F. Solgun, New J. Phys. 15, 075001 (2013).

[15] S. B. Bravyi and A. Y. Kitaev, arXiv:quant-ph/9811052; R. Raussendorf and J. Harrington, Phys. Rev. Lett. 98, 190504 (2007).

[16] A. G. Fowler, M. Mariantoni, J. M. Martinis, and A. N. Cleland, Phys. Rev. A 86, 032324 (2012).

[17] J. T. Barreiro, M. Müller, P. Schindler, D. Nigg, T. Monz, M. Chwalla, M. Hennrich, C.F. Roos, P. Zoller, and Rainer Blatt, Nature (London) 470, 486 (2011).

[18] W. Pfaff, T. H. Taminiau, L. Robledo, H. Bernien, M. Markham, D. J. Twitchen, and R. Hanson, Nat. Phys. 9, 29 (2013).

[19] D. Ristè, M. Dukalski, C. A. Watson, G. de Lange, M. J. Tiggelman, Y. M. Blanter, K. W. Lehnert, R. N. Schouten, and L. DiCarlo, Nature (London) 502, 350 (2013).
[20] A. Wallraff, D. I. Schuster, A. Blais, L. Frunzio, R.-S. Huang, J. Majer, S. Kumar, S. M. Girvin, and R. J. Schoelkopf, Nature (London) 431, 162 (2004).

[21] J. P. Groen, D. Ristè, L. Tornberg, J. Cramer, P. C. de Groot, T. Picot, G. Johansson, and L. DiCarlo, Phys. Rev. Lett. 111, 090506 (2013).

[22] M. Jerger, S. Poletto, P. Macha, U. Hbner, E. Ilichev, and A. V. Ustinov, Appl. Phys. Lett. 101, 042604 (2012).

[23] Y. Chen et al., Appl. Phys. Lett. 101, 182601 (2012).

[24] G. Haack, F. Helmer, M. Mariantoni, F. Marquardt, and E. Solano, Phys. Rev. B 82, 024514 (2010).

[25] M. Mariantoni et al., Science 334, 61 (2011).

[26] J. Johansson, S. Saito, T. Meno, H. Nakano, M. Ueda, K. Semba, and H. Takayanagi, Phys. Rev. Lett. 96, 127006 (2006).

[27] F. W. Strauch, P. R. Johnson, A. J. Dragt, C. J. Lobb, J. R. Anderson, and F. C. Wellstood, Phys. Rev. Lett. 91, 167005 (2003).

[28] See Supplemental Material at http://link.aps.org/ supplemental/10.1103/PhysRevLett.112.070502 for experimental methods, extended results, and supporting theory.

[29] J. E. Johnson, C. Macklin, D. H. Slichter, R. Vijay, E. B. Weingarten, J. Clarke, and I. Siddiqi, Phys. Rev. Lett. 109, 050506 (2012); D. Ristè, J. G. van Leeuwen, H.-S. Ku, K. W. Lehnert, and L. DiCarlo, Phys. Rev. Lett. 109, 050507 (2012).

[30] We were unable to tune the JPA resonance frequency high enough to align with $A$ 's resonator, which limited smallsignal gain to $4.2 \mathrm{~dB}$.

[31] D. I. Schuster, A. Wallraff, A. Blais, L. Frunzio, R.-S. Huang, J. Majer, S. M. Girvin, and R. J. Schoelkopf, Phys. Rev. Lett. 94, 123602 (2005).

[32] The population transfer fidelity was limited by a spurious resonance $600 \mathrm{MHz}$ above the bus resonator and strongly coupled to $A$.

[33] W. K. Wootters, Phys. Rev. Lett. 80, 2245 (1998).

[34] N. S. Williams and A. N. Jordan, Phys. Rev. A 78, 062322 (2008).

[35] R. Ionicioiu, Phys. Rev. A 75, 032339 (2007).

[36] L. Steffen, Y. Salathe, M. Oppliger, P. Kurpiers, M. Baur, C. Lang, C. Eichler, G. Puebla-Hellmann, A. Fedorov, and A. Wallraff, Nature (London) 500, 319 (2013).

[37] Z. Chen et al., arXiv:1310.2325.

[38] Chow et al., arXiv:1311.6330. 